\title{
Frantically forging fermium
}

\author{
Brett F. Thornton and Shawn C. Burdette relate how element 100 was first identified in a nuclear weapons \\ test, but that was classified information, so researchers had to 'discover' it again using other methods.
}

W hen the circumstances surrounding a discovery mean that its publication is blocked by external forces, how do you ensure that you can claim appropriate credit for your findings? This was the case with the discovery of element 100 - fermium which was named for Enrico Fermi who, among many other things, developed the first nuclear reactor. Fermium was first produced in the Ivy Mike thermonuclear weapons test at Enewetak Atoll in November 1952; this led to a rush to produce the element by different means in order to circumvent a publication embargo on results associated with the tests on that group of islands in the Pacific Ocean.

Undiscovered heavy transuranium isotopes had been postulated as by-products of large nuclear explosions because the high neutron flux might cause uranium to undergo multiple neutron captures almost instantaneously. A research group led by Albert Ghiorso at the University of California Radiation Laboratory (UCRL) found element 99 in atmospheric filter samples collected from the Ivy Mike test. In larger fallout samples from a nearby atoll they found several other neutron-rich isotopes, including ${ }^{255} 100$. The formation of ${ }^{255} 100$ was attributed to the $\beta$-decay of ${ }^{255} 99$, which itself originated from multiple $\beta$ decays of uranium that had absorbed many neutrons in the explosion to yield ${ }^{255} \mathrm{U}$. ${ }^{255} 100$ eluted from an ion-exchange column at the expected time ${ }^{1,2}$; however, the UCRL team was barred from reporting a discovery from the classified weapons test. Ghiorso knew his team had discovered element 100 , but fretted about losing credit if scientists elsewhere produced the element independently and published first.

Ghiorso's concerns were valid. At the Nobel Institute of Physics (NI) in Stockholm, a cyclotron had been recently upgraded to produce heavy-ion beams ${ }^{3}$. On 19 February 1954, NI scientists produced ${ }^{250} 100$ by

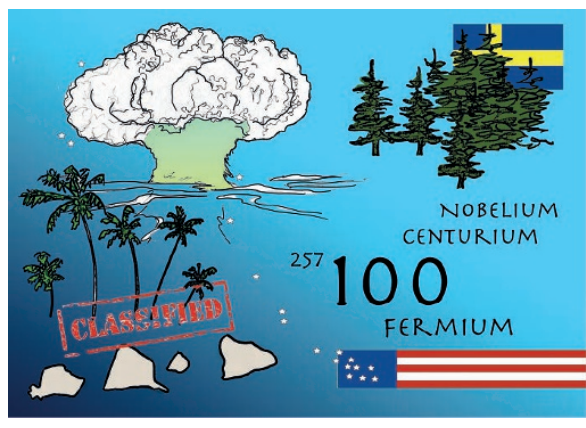

bombarding a uranium target with ${ }^{16} \mathrm{O}^{6+}$ ions for several hours ${ }^{4}$. Due to the numerological significance, the name centurium for element 100 had been circulating in the scientific community. NI director Manne Siegbahn wrote to Glenn Seaborg at UCRL to inform him of the discovery and suggested the name nobelium.

The UCRL group, realizing they were in a discovery race, had been conducting similar experiments with oxygen- and nitrogen-ion beams on uranium and plutonium targets. After producing ${ }^{253} 99$, the UCRL team coaxed another neutron into the nucleus, hoping to obtain ${ }^{254} 99$, which would then undergo $\beta$-decay to form ${ }^{254} 100$. Their success was published ${ }^{5}$ on 1 March 1954 - a mere ten days after the production of ${ }^{250} 100$ in Stockholm - in a paper in which they took care to overtly mention the existence of earlier "unpublished [classified] information". The NI article would appear just a few months later, on 15 July $^{4}$.

The earlier classified information - the production of element 100 in the Ivy Mike test - was not publicly reported until June 1955 in a joint paper by UCRL, Argonne National Laboratory and Los Alamos Scientific Laboratory, the last of which was credited with building the weapon ${ }^{2}$. In the paper, the UCRL group unequivocally argued this earlier discovery in fallout should take precedence. The first sentence of the paper makes that claim crystal clear: "This communication is a description of the results of experiments performed in
December, 1952 and the following months". To cement their discovery priority, they suggested the name fermium in the paper's title.

Typically in science, two independent, near-simultaneous discoveries are afforded recognition. Oxygen was independently found by Joseph Prisetley in 1774 and Carl Wilhelm Scheele around 1773. Lutetium was independently discovered by Georges Urbain and Carl Auer von Welsbach in 1907. Nevertheless, the Stockholm group is rarely, if ever, credited with a shared discovery of element 100 . The Stockholm experiments reported in 1954 probably represent an independent discovery of element 100: with Cold War secrecy, the NI group was unlikely to have known about the UCRL group's earlier discovery before its publication in 1955 .

Element 100 was not the last bit of element-discovery controversy for the NI. A few years later, they reported the production of element 102, a claim challenged by both UCRL and a Soviet group. Assigning discovery credit of element 102 took decades. This time, however, the name provided for element 102 by the NI group - nobelium - stuck, even though their discovery claim did not ${ }^{6}$.

BRETT F. THORNTON is in the Department of Geological Sciences and Bolin Centre for Climate Research, Stockholm University, 10691 Stockholm, Sweden.

e-mail: brett.thornton@geo.su.se

\section{SHAWN C. BURDETTE is in the}

Department of Chemistry and Biochemistry, Worcester Polytechnic Institute, Worcester, Massachusetts 01609-2280, USA.

e-mail: scburdette@WPI.EDU

\footnotetext{
References

1. Ghiorso, A. Chem. Eng. News. 81, 174-175 (2003).

2. Ghiorso, A. et al. Phys. Rev. 99, 1048-1049 (1955).

3. Atterling, H. Arkiv Fysik. 7, 503-506 (1954).

4. Atterling, H., Forsling, W., Holm, L. W., Melander, L. \& Åström, B. Phys. Rev. 95, 585-586 (1954).

5. Harvey, B. G., Thompson, S. G., Ghiorso, A. \& Choppin, G. R. Phys. Rev. 93, 1129 (1954).

6. Thornton, B. F. \& Burdette, S. C. Nat. Chem. 6, 652 (2014).
} 